\title{
10. Risk Responses, Emergency Management and Community Resilience in the Aftermath of the Recent Victorian Natural Disasters
}

\author{
Neil Comrie
}

This contribution focuses on risk responses, emergency management and the development of community resilience in the context of two natural disasters in Australia: the 2009 Victorian bushfires and the 2010 Victorian floods. My contribution arises from experience as a police officer of 35 years' service, including eight years as chief commissioner of Victoria Police and on-theground practical experience; also relevant is my experience of reviewing the 67 recommendations arising out of the 2009 Victorian Bushfires Royal Commission, and with reviewing the 2010 Victorian floods. From my perspective, I would argue that in recent years our capacity to deal with natural disasters has been tested more than at any point in the history of the Australian nation; however, while natural disasters are damaging events, they also provide an opportunity to learn.

Following these two major natural disasters in Victoria - the bushfires and the floods - there has been some critical analysis of our preparation and planning before the disasters and our subsequent responses to them and recovery. Consequently, we now have a substantial body of recent evidence we can use to inform our decision-making for the future. And there are two broad conclusions we can draw from the many reviews that have been conducted: first and foremost, we cannot always prevent or avoid major emergencies; and second, we realise they will continue to occur in the future. We can, however, reduce the risk and mitigate the damage of these emergencies through improved planning, preparation and coordination of response. And an important lesson that comes out of the two major events in Victoria is that the State Government must overhaul the flawed approach to managing emergency resources that has prevailed across all relevant agencies.

My most significant argument here, however, is that our communities must be equipped to be better able to survive and recover from disasters. Changes in legislative policy processes are needed, but it is clear that the most important improvement we can make to existing emergency management is to ensure our communities are more capable of looking after themselves. I will try to explain the rationale for these comments below. 
Many lessons are to be learnt from the 2009 Victorian Bushfires Royal Commission. The most important issue to arise from the royal commission is the need for everyone in the community, from the individual right through to government, to play a role and take a share of the responsibility for dealing with emergencies. We have known for some time that probably the best means of emergency prevention is the education of adults and children. In fact, it was suggested some decades ago that fire prevention be made a part of the curriculum in every school. This was the finding of the Stretton Royal Commission after the 1939 Black Friday bushfires, in which 71 Victorians lost their lives. Now 73 years after this recommendation was made, and after the tragedy of the 2009 fires has been digested, it appears that education about bushfire safety will finally be included in the national curriculum.

I undertook the Victorian Floods Review in 2011, which turned into a protracted investigation into the factors associated with the floods. A large body of evidence was gathered from extensive community consultations. We visited all of the flood-affected areas in Victoria, held public meetings, spoke to those people directly affected by the floods, met with the local governments in those areas, received 150 written submissions, and undertook operational debriefings with all of the agencies involved in responding to the floods and which had extensive consultation with stakeholder agencies. Out of all of these consultations, the major issue of concern that emerged was that our communities were largely unaware of the risks they faced; consequently, they were ill prepared and incapable of looking after themselves in the face of adversity.

Evidence of a lack of community resilience included a poor understanding of their risk environments, despite the fact that people were living on floodplains. In many cases, they had no idea they were at risk of flood. Hospitals and community centres were built on floodplains and, as with the hospital at Charlton, were basically destroyed by the flood. Moreover, flood-mitigation plans were inadequate: people living in those locations were given no prior warning by the authorities that they lived on floodplains. There was little local planning or preparation for a response to an event of that nature. The structures for local responses were poor: they were disorganised and therefore extremely ad hoc. Two findings from the consultations were particular to some smaller, often isolated towns. First, emergency services found it difficult to access these areas. Second, emergency services personnel who happened to reside in the towns were forced to divide their attention between looking after their own properties and families on the one hand and trying to do their job on the other. The reality was that because of this isolation most people had no real idea of what they should do to look after themselves.

People did not know where to go for advice and knowledge about what should happen, and in many instances they simply were unaware of who was in 
charge and who was able to give directions. There was also poor capacity for mitigating the flood once it struck. In some cases, elementary procedures were adequate, but many people did not know how to fill sandbags or how to stack them. Despite their best efforts, in a lot of instances when the sandbags were placed in rows, they were washed away because they were not placed properly. Simple processes such as sandbagging often go unnoticed by policymakers, but the Victorian floods demonstrate that we must consider the need to provide education in the basics of disaster mitigation.

There was also confusion resulting from inaccurate and untimely warnings. A new facility called Emergency Alert, a telephone-based warning system that sends SMS information messages to local residents, was not in place for the Black Saturday bushfires of February 2009, but it was for the 2010 floods. Yet, emergency managers still had a lot of work to do because in a number of instances the warnings sent out were unclear and confusing. In some instances people were not sure whether information was a warning or simply advice; they became confused about what it was they were being told. Perhaps the most concerning aspect, from my perspective, was that many people were completely reliant on the emergency service organisations to give them some support and direction when they found themselves in trouble; successful disaster management requires that people who are threatened by the disaster have some autonomous capacity to resist its effects.

So what do we make of that lack of resilience and why has it occurred? The evidence indicates that many people felt they had been completely disempowered from the process of looking after themselves in an emergency, that the Government had centralised all the resources and the authority to deal with those situations. This created an expectation that, in the face of adversity, the State authorities would save residents - even at the last moment. Many of the respondents we spoke to in the community said the State Government had dictated to communities on emergency management rather than working with communities on such management. There is absolutely no doubt that apathy and complacency had developed in the communities affected by the Black Saturday bushfires and the Victorian floods. One relevant example about community apathy was demonstrated 12 months after the Black Saturday bushfires in Victoria, when the Country Fire Authority of Victoria (CFA) conducted a survey of communities affected by bushfires. Even though 173 people had lost their lives in this disaster, 80 per cent of the respondents to the survey said they would still wait to see whether there was smoke and fire on the horizon before they left their homes - by which time it is too late to leave. We have a serious problem trying to deal with community apathy. And the real problem is that the 
more government does for people, the more they expect of it. Government has created and encouraged a relationship in which the community is dependent on it and is unable to fend for itself.

After the review of the Victorian floods, one of the recommendations I made in the report was that Victoria should establish a network of community resilience committees to develop and administer community resilience plans based on an 'all hazards' approach and tailored to the specific needs of each community.

The 'all hazards, all agencies' maxim is one of the core issues Victoria needs to address. The term means that a flexible integrated arrangement is in place between the relevant emergency agencies so that an integrated response is forthcoming regardless of the agency emergency officers serve in or the particular hazard they are confronting. In Victoria we are going through a major reform program because some of the inherited shortcomings of the previous emergency response regime have been identified. The legislation in Victoria for the Metropolitan Fire Brigade and the CFA was created in 1958. Apart from a few adjustments, the legislation clearly reflects its age. It created a set of silo structures where the critical firefighting agencies each have separate boards; from a governance viewpoint they are required to make sure the organisations deliver against the legislation, but they can do so with little regard for agencies in neighbouring areas or other sectors. Ideally, when faced with major disaster events such as widespread bushfires or floods, all the emergency services agencies, irrespective of their primary role and responsibility, have to come together and work as a collective; however, with separate, disparate communication systems, different equipment that is not interchangeable and with cultures that are quite different, a range of barriers prevents the concept of cooperative response. I hope the ongoing review of the legislation, including a white paper consultation process, will provide some overarching policy and structure that will drive that sort of philosophy.

More importantly, one of the key things is to train our people in an environment in which they understand each others' roles and are trained to work together from the outset. Let me use the examples of the police and military services, although similar examples are found in most of the emergency professions. To become a police officer or a soldier, basic training equips the new trainees to do the basic functions in those services. Afterwards, as officers, they specialise in whatever it might be, perhaps detective work or the artillery or as engineers. All emergency management workers should go through the same fundamental training and emergency management and then specialise in a firefighting team or a State emergency service. That should do two things: it would ensure shared consistent training at the fundamental level; and it would act as a barrier to some of the distinctive cultural problems that exist and inevitably develop over time. So when people come together in a major event they already know each other because they have trained together, and the cultural barriers disappear quickly in that environment. 
Shared basic training does not necessarily mean, however, identical arrangements across all towns and regions. One of the issues that became apparent when we inspected the sites of the floods and talked to communities was that a one-sizefits-all plan is not feasible. And so I recommended that the emergency service organisations should be required to consult and engage with local community resilience committees in the preparation, planning, response and recovery phases of emergency management.

It was interesting that while we were doing the flood review, the Council of Australian Governments (COAG) released the National Strategy for Disaster Resilience, which reiterated many recommendations about shared responsibility made by the bushfire royal commission.

In undertaking research and compiling our report on the flood disaster, it became evident that the idea of community resilience was not a new one-resilience models have been established around the world, including in New Zealand, Canada, the United Kingdom and the United States. These models inform the community that immediate assistance by emergency service responders may not be readily available and that they should be prepared and able to cope on their own for up to three days. All these models include information on understanding the risk that natural disasters pose to the community, on making an emergency plan and assembling an emergency supply kit. One example that prevails in New Zealand schools is that the threat of earthquakes is a dedicated topic addressed early in the curriculum. It is not clear why such models have not been replicated elsewhere.

Further, during the course of our investigative review, the Queensland Government, which had managed its own emergency response to Cyclone Yasi at the same time as the Victorian floods, launched a new website called 'Harden Up Queensland'. On the front page of that website is the following statement: 'Weather events are getting more severe and when a major weather event hits you cannot rely on government and volunteer organisations to help. You need to harden up by preparation, awareness and helping others.'

That was a courageous stance for the Government to adopt because, prior to that in Australia, a 'softly, softly' approach to informing people of their responsibilities and power to resist disasters had prevailed. The Bligh Queensland Government was really the first State government I had seen stating directly to people that they had to take some responsibility of their own during natural disasters.

Across Australasia we must now formulate a multi-pronged approach to achieving community resilience to natural disasters. The first step is for governments and emergency service organisations to realise that they cannot protect our communities in all circumstances. We have to disabuse people of the 
notion that emergency service organisations will save them at the last moment; to pretend otherwise is not only deceptive but also dangerous, because it will cause people to become complacent and stay in their homes until it is far too late to evacuate before the oncoming disaster. The royal commission into the Black Saturday bushfires found that in several cases people died because they stayed in their homes too long. We must also ensure that our communities are informed of the characteristics and capacity of their emergency services. For example, it would be worth publicising that in regional Victoria, more than 90 per cent of our emergency service organisations are staffed by volunteers who have other responsibilities.

Governments must also invest in good forward planning, which should include specific, practical, risk-related policies that can be implemented to reduce loss of life and community damage. We need realistic integrated flood plans that are based not on artificial municipal boundaries but on the footprint of the floodplain itself. Floods ignore artificial jurisdictional boundaries. Our State and municipal authorities need to revisit their flood-mitigation plans. We also need to plan infrastructure with the risk of flooding in mind. One of the problems here is that our forebears deliberately built along rivers so they could have access to water. Towns lie on floodplains; farmers farm on floodplains for the fertile soil. Hence, we cannot claim that all the consequent risks can be eliminated, but if the areas of highest risk are identified this can be built into future planning strategies to deal with them.

In relation to the risk of further fires, we need to better use our existing infrastructure. The Fire Services Commissioner in Victoria appointed after the bushfire royal commission is now looking at a strategy in which all public buildings in fire-prone areas such as schools and public halls in the future will be built to a specified standard, permitting them to be used as community fire refuges. Because of the high cost of retrofitting buildings or trying to adapt buildings in high-risk areas the Victorian Government decided to buy back some land where homes were burnt and other properties damaged; this is to prevent rebuilding in fire-prone areas. The central authorities are working through this process, but some councils have other pressing needs and agendas, including a rate base that they are trying to protect. In a couple of instances, new estates are being developed almost adjacent to those areas where the Government is buying back land - a truly bizarre phenomenon. Strong decision-making must come to the fore; retrospective correction is very difficult but taking a hard line and making decisions to ensure we do not repeat those mistakes again are necessary to reduce the chances of future disasters.

Another important element in community resilience will be to include private enterprises in planning. There is a real challenge here for communities to pick up where they have privately owned infrastructure that impacts on the wellbeing of 
the whole community. In Victoria the owner of an electricity substation located on a floodplain had no protective measures in place to keep electricity flowing to the community. That is why it is important for the community resilience strategy to identify such potential risks (even when it involves private installations) and to try to put some strategies in place to plan for them in ways that involve not exclude the private sector. My assessment is that if the private sector is engaged early enough and its members understand the community priorities they tend to become more involved more quickly than if they just see it as an inconvenient cost factor that will disappear in due course. It is just part of that process of involving every stakeholder in the planning process so that communities can have contingencies in place should an event arise.

We also have to make a long-term commitment to address the apathetic culture demonstrated by many in our communities and raise the level of awareness of their role in combating natural disasters. In other policy areas we have been able to do this. For instance, significant cultural change has taken place on Australian roads through things such as the Victorian Transport Accident Commission advertisements, which have been major contributors to the reduction in the road toll; and the QUIT campaign has seen a significant reduction in cigarette smoking throughout Australia. Long-term social education programs like these are critical to developing a strong sense of community resilience for natural disasters. We need similar campaigns to focus on community preparedness.

I have argued strongly that I do not think we need to create a whole new separate community structure to achieve this, because in every country town in Victoria there are pre-existing community service organisations looking for opportunities to serve their community. Lions clubs, Rotary clubs and Country Women's Association branches are among a wide range of organisations which, if appropriately engaged, would be a powerful force in building this community resilience.

We need to facilitate the education of communities so we can empower them. We need to work in conjunction with them, rather than directing them when disasters strike. We need to encourage communities to discover their own learning about resilience in preparation for natural disasters. Moreover, local governments must play a much more active role in emergency management than they have in the past - this is certainly the lesson from the Victorian natural disasters.

In conclusion, we still need to make a whole range of structural policy and legislative changes. In Victoria the Government has started to do this by commissioning various green and white policy papers on this issue. These will propose a range of options for the development of a new emergency management 
environment. In my view, the most effective and significant improvement in emergency management will flow from strong and resilient communities that are not absolutely dependent on emergency services at times of crisis.

Communities that have involvement in and ownership of plans for their own safety have a much greater capacity to look after themselves. Moreover, this actually presents governments with additional opportunities to use those arrangements for other community capacity building. In Australia over the past few decades, a lot of social and community structures that existed in small country towns have declined. This absence of social capital militates against governments effectively communicating with local communities. Reflecting on the aftermath of disasters, like the Victorian fires and floods, and thinking about future emergency management systems provide an opportunity to build those community social structures again.

The major challenge for government and its agencies, however, is to have the courage to relinquish the long-established practice of central control of emergency management and to devolve it to local communities. For some that will be a very difficult challenge, but from the Victorian perspective it is obvious that maintenance of the status quo is not a viable option, for as Margaret Mead once said: 'Never doubt that a small group of thoughtful concerned citizens can change the world. Indeed it's the only thing that ever has.' 
This text taken from Future-Proofing the State: Managing Risks, Responding to Crises and Building Resilience, edited by Jonathan Boston, John Wanna, Vic Lipski and Justin Pritchard, published May 2014 by ANU Press, The Australian National University, Canberra, Australia. 